\title{
Background-Free Nonlinear Microspectroscopy with Vibrational Molecular Interferometry
}

\author{
Erik T. Garbacik ${ }^{1}$, Jeroen P. Korterik ${ }^{1}$, Cees Otto ${ }^{2}$, Shaul Mukamel ${ }^{3}$, Jennifer L. Herek ${ }^{1}$, and \\ Herman L. Offerhaus ${ }^{1, *}$
}

${ }^{1}$ Optical Sciences Group, MESA+ Institute for Nanotechnology, University of Twente, 7500AE Enschede, The Netherlands ${ }^{2}$ Medical Cell BioPhysics Group, MIRA Institute for Biomedical Technology and Technical Medicine, University of Twente, 7500AE Enschede, The Netherlands ${ }^{3}$ Department of Chemistry, University of California, Irvine, California 92697, USA

\begin{abstract}
We demonstrate a method for performing nonlinear microspectroscopy that provides an intuitive and unified description of the various signal contributions, and allows the direct extraction of the vibrational response. Three optical fields create a pair of Stokes Raman pathways that interfere in the same vibrational state. Frequency modulating one of the fields leads to amplitude modulations on all of the fields. This vibrational molecular interferometry technique allows imaging at high speed free of nonresonant background, and is able to distinguish between electronic and vibrational contributions to the total signal.
\end{abstract}

For a number of decades much of the development of new coherent anti-Stokes Raman scattering (CARS) techniques has been focused on suppressing or eliminating the persistent nonresonant background that reduces contrast and can render experiments involving low concentrations of resonant oscillators impossible. Various methods developed so far include exploiting the polarization dependences of the resonant and nonresonant components of $\chi^{(3)}$ [1-5], directly measuring [6-8] or extracting [9-11] the vibrational phase of the oscillators, shaping the phase of a broadband optical pulse to match that of the molecule [12-15], or introducing temporal delays to probe the resonant vibrational state after the nonresonant coherence has decayed $[16,17]$.

Recent work by Rahav and Mukamel [18] introduced a new paradigm regarding coherent Raman scattering experiments. Rather than operating in the common semiclassical field perspective, they focus on energy transfer from a molecular quantum mechanical point of view. The semiclassical approach of nonlinear optics assumes classical fields interacting with quantum matter. The detected mode is singled out from the outset and is described using the macroscopic Maxwell's equations. Heterodyne detection is viewed as an interference of the signal field with a local oscillator field, which makes it hard to establish connections between different experiments with the same pulse configuration where different modes are detected. The quantum description of heterodyne-detected four-wave mixing is much more transparent. We consider a steady state of the molecule with ground state $|a\rangle$ and vibrational state $|c\rangle$, and four modes of the radiation field $\left(\omega_{1}-\omega_{2}=\omega_{4}-\omega_{3}=\right.$ $\omega_{c a}$, with $\omega_{2}<\omega_{1}$ and $\omega_{3}<\omega_{4}$ ). The optical field modes are all far detuned from the lowest electronic excited state $|b\rangle$. All modes, including the local oscillator, are treated in the same

(C) 2011 American Physical Society

*To whom all correspondence should be addressed. h.l.offerhaus@utwente.nl. 
microscopic way. Heterodyne detection then emerges as a stimulated process involving the detected mode. This approach provides a more intuitive and unified description of the various signals and traces their microscopic origins.

The probability for a Raman-active transition from the ground state $|a\rangle$ to a vibrational state $\mid$ $c\rangle$ is

$$
P_{a \rightarrow c}=P_{a \rightarrow c}^{12}+P_{a \rightarrow c}^{34}+P_{a \rightarrow c}^{1234}
$$

where the terms $P_{a \rightarrow c}^{12}$ and $P_{a \rightarrow c}^{34}$ are the individual pump-probe (Stokes Raman) processes into the vibrational state. The last term, $P_{a \rightarrow c}^{1234}$, is the interference of these two processes that yields the resonant component of the CARS signal, and is associated with the imaginary component of $\chi^{(3)}$. This resonant dissipative term involves energy that is transferred from the optical fields into the molecule. In addition to this dissipative term there is a nonresonant parametric component $S^{\text {par }}$ that is equivalent to the real part of $\chi^{(3)}$ in which energy is merely rearranged between the field modes and the molecule returns to the ground state. The parametric and dissipative energy level diagrams are shown in Figs. 1(a) and 1(b), respectively.

An emission process produces an increase in the intensity of a given field mode, whereas an absorption process results in a decrease in intensity. By tallying the gain and loss contributions from the dissipative and parametric processes for each field mode, we find that the changes in the intensities of the field modes after interacting with a sample are then given by

$$
S_{1}=-\frac{1}{2} P_{a \rightarrow c}^{1234}-S^{\mathrm{par}}, S_{2}=+\frac{1}{2} P_{a \rightarrow c}^{1234}+S^{\mathrm{par}}, S_{3}=+\frac{1}{2} P_{a \rightarrow c}^{1234}-S^{\mathrm{par}}, S_{4}=-\frac{1}{2} P_{a \rightarrow c}^{1234}+S^{\mathrm{par}},
$$

where the factor of $1 / 2$ signifies that only one of the Stokes Raman processes affects the number of photons in each field mode. From these relations it is clear that the parametric contribution may be eliminated by measuring $S_{4}-S_{2}=-P_{a \rightarrow c}^{1234}$, which is the purely dissipative interference term. In this Letter we demonstrate the measurement of this purely dissipative signal.

The magnitude of the dissipative energy transfer is very small compared to the incident field amplitudes $\left(\delta I / I<10^{-4}\right)$. To separate this signal from the large dc background we shift the frequencies of the two Stokes Raman pathways $(1 \rightarrow 2$ and $4 \rightarrow 3)$ relative to each other. The population in the vibrational level is modulated by the beating of these two pathways, and the modulation carries over onto each of the driving fields as an amplitude fluctuation at the difference frequency. This amplitude modulation is then detected on each field separately using lock-in amplification. Rather than using four independent fields, we synchronously pump an optical parametric oscillator (OPO) with the second harmonic of a 1064-nm laser [see Fig. 1(left)] to generate a pair of frequency-locked beams, the signal and idler. The frequency of the residual laser fundamental is shifted with an acousto-optic modulator (AOM). All three beams are subsequently mixed in the sample. The vibrational frequency accessed by the combination of the laser fundamental $\left(S_{1}\right)$ and idler $\left(S_{2}\right)$ is identical to that of the laser fundamental $\left(S_{3}\right)$ and signal $\left(S_{4}\right)$; in the former situation, the laser fundamental is used as a pump beam, while in the latter it functions as the Stokes. Because the laser fundamental is used in opposite ways in the two Stokes Raman processes, the difference frequency between the two pathways is twice the frequency applied to the AOM. All of the beams carry an amplitude modulation at the beat frequency of these two 
Stokes Raman pathways, and their relative signs indicate whether a net gain or loss is observed: the signal experiences loss and the idler gain, while the gain or loss of the laser fundamental is determined by which Stokes Raman pathway is dominant. In our experiment the signal field is stronger than that of the idler, and so the laser fundamental field carries net gain in the presence of a vibrational state.

Interestingly, electronic states resonant with the two-photon absorption of the laser fundamental second harmonic and the signal-idler sum frequency can also generate the amplitude modulations as described above [Fig. 1(c)]. However, the idler and the laser fundamental experience net gain in a vibrational resonance, whereas all fields experience net loss in a resonant electronic transition. The difference of the idler $\left(S_{2}\right)$ and signal $\left(S_{4}\right)$ intensities contains no electronic contribution, while the laser fundamental experiences loss in an electronic level and, as stated above, gain in a vibrational level. Monitoring the relative gain and loss of all three beams therefore allows us to distinguish between electronic and vibrational resonances without interference from nonresonant back-ground. We refer to this process as vibrational molecular interferometry, or VMI.

The optical setup used for these experiments is similar to that described by Jurna et al [19]. A frequency-doubled Nd: $\mathrm{YVO}_{4}$ laser (Coherent Paladin) pumping an optical parametric oscillator (APE Berlin Levante Emerald) generates three frequency- and phase-locked beams. An acousto-optic modulator (AOM) placed in the laser fundamental beam shifts the carrier frequency of that beam by $500 \mathrm{kHz}$. The three beams are expanded with telescopes, temporally overlapped with delay stages, and spatially combined on a pair of dichroic mirrors. The maximum average power on the sample is about $130 \mathrm{~mW}$ ( $80 \mathrm{~mW}$ signal, 30 $\mathrm{mW}$ laser fundamental, $20 \mathrm{~mW}$ idler) and decreases as the OPO is tuned away from its gain optimum. The idler is set to be slightly convergent to compensate for chromatic aberration of the focusing objective. Wave plates in each beam are used to align all polarizations along the same direction. The beams are laterally scanned with a pair of galvano mirrors (Olympus FluoView300/IX71), focused into the sample with a 1.2 NA water immersion objective (Olympus UPLSAPO), collected in the forward direction with a 0.55 NA long-workingdistance objective, and spectrally separated onto individual detectors with dichroic mirrors. The idler beam is detected on a large-area InGaAs photodiode (ThorLabs FGA21), while the laser fundamental and signal beams are each sent to separate large-area silicon diodes (ThorLabs TDS1010). Forward- and backward-scattered CARS and fluorescence emissions are transmitted through spectral bandpass filters centered at the CARS wavelength and detected on photo-multiplier tubes (Hamamatsu R3986). The outputs of all four forward detectors are sent to a pair of high-frequency lock-in amplifiers (Zurich Instruments HF2-LI) set to de-modulate the second harmonic of the modulation frequency on the laser fundamental. Only the amplitude components of these signals are used in the VMI experiment.

For microscopy on a sample of mayonnaise the output of the OPO is fixed to probe the symmetric $\mathrm{CH}_{2}$ stretch at $2845 \mathrm{~cm}^{-1}\left(\lambda_{s}=816.8 \mathrm{~nm}, \lambda_{i}=1526 \mathrm{~nm}\right)$, and the beams are raster scanned across the sample. An image containing $256 \times 256$ pixels is acquired without averaging in about $4 \mathrm{~s}$ with a 25 - $\mu$ s lock-in time constant. The amplitudes of the signal and idler channels are corrected for differences in the spectral responses of the detectors, scaled to account for the lock-in detector settings, and subtracted from each other to produce a background-free image $\left(S_{4}-S_{2}\right)$ in real time. Figure 2 demonstrates the comparison of forward-detected CARS, background-free vibrational phase contrast (VPC) CARS [19], and VMI. A strong nonresonant background from water in the sample significantly reduces contrast in the F-CARS image, but is absent in both the VPC-CARS and VMI images. Differences between the VMI and VPC-CARS images are attributed to the lack of a phasematching condition in the former [18]. 
Background-free spectroscopy with VMI is demonstrated in the alkyl region on a sample of neat dimethyl sulfoxide (DMSO), and compared to CARS and VPC-CARS spectra in Fig. 3. The overall agreement between the two background-free techniques is good over most of the spectrum. In particular, the features of the CARS spectrum due to the nonresonant background-a shift of the main peak (nominally $2915 \mathrm{~cm}^{-1}$ ) to a lower frequency (2912 $\mathrm{cm}^{-1}$ ), marked asymmetry of that prominent peak, and a skewed ratio of heights of the 2915- $\mathrm{cm}^{-1}$ and $3000-\mathrm{cm}^{-1}$ peaks - are noticeably absent in both the VPC-CARS and VMI spectra. Low optical power on the edge of the OPO gain curve contributes to noise in the VMI measurement that manifests as an offset between the VMI and VPC-CARS spectra below $2905 \mathrm{~cm}^{-1}$. Note that this offset does not appear in the VMI measurement shown in Fig. 4(b).

Electronically resonant processes can present problems for CARS and spontaneous Raman scattering measurements because of fluorescent emissions. As a model example of a problematic system, we use DCM-pyran, a laser dye which has a broad absorption band covering most two-photon resonances of the wavelengths used in this experiment, and an emission maximum near the CARS wavelength [see Fig. 4(a) for spectra] [20]. The emission spectrum from a saturated solution of DCM-pyran in DMSO is dominated by fluorescence, masking the strong $2912-\mathrm{cm}^{-1}$ resonance in the backward-scattered CARS signal, as shown in Fig. 4(b). However, the VMI spectrum (signal minus idler) clearly shows the backgroundfree DMSO peak with only a minor residual contribution from the DCM-pyran.

Furthermore, the modulation detected on the laser fundamental [labeled "1064 nm" in Fig. 4(b)] shows net loss from the electronic DCM-pyran contribution away from the DMSO resonance, and a positive peak at the DMSO resonance.

In summary, we have demonstrated a new quantum mechanical approach for nonlinear microspectroscopy that exploits interference between two competing Stokes Raman pathways in analogy to coherent control [21,22]. Wave mixing techniques such as CARS which only detect a single beam contain nondissipative (parametric) contributions that reflect energy exchange between field modes and add undesired, matter-independent background. However, with this more elaborate detection of all modes we have shown that it is possible to convert CARS into a fully dissipative technique. Compared to the more practical technique of stimulated Raman scattering (SRS), VMI has the potential to provide more insights at the cost of technical complexity. For example, the vibrational phase can be retrieved (not shown here) so that mixtures with overlapping resonances can be analyzed [8]. The use of properly phased broadband pulses, akin to femtosecond stimulated Raman scattering (FSRS) techniques $[23,24]$, would produce stronger signals than are currently obtained in narrowband VMI [25].

\section{Acknowledgments}

We are grateful for many useful discussions with Alexander van Rhijn, Dr. Chris Lee, and Dr. Pepijn Pinkse. Partial funding is provided by the Stichting voor Fundamenteel Onderzoek der Materie (FOM), and by a VICI grant to J. L. H. from the Nederlandse Organisatie voor Wetenschappelijk Onderzoek (NWO). S. M. gratefully acknowledges the support of the National Institutes of Health (Grants No. GM059230 and No. GM091364), NSF Grant No. CHE-1058791, and DARPA BAA-10-40 QUBE. We further thank Coherent, Inc. for use of the Paladin laser, and APE Berlin for the Levante Emerald OPO.

\section{References}

1. Akhmanov SA, Bunkin AF, Ivanov SG, Koroteev NI. JETP Lett. 1977; 25:416.

2. Chikishev A, Lucassen G, Koroteev N, Otto C, Greve J. Biophys. J. 1992; 63:976. [PubMed: 1330043]

3. Lucassen, G. Ph.D. thesis. University of Twente; 1992. 
4. Cheng J-X, Book LD, Xie XS. Opt. Lett. 2001; 26:1341. [PubMed: 18049602]

5. Orsel K, Garbacik ET, Jurna M, Korterik JP, Otto C, Herek JL, Offerhaus HL. J. Raman Spectrosc. 2010; 41:1678.

6. Potma EO, Evans CL, Xie XS. Opt. Lett. 2006; 31:241. [PubMed: 16441043]

7. Jurna M, Korterik JP, Otto C, Herek JL, Offerhaus HL. Opt. Express. 2008; 16:15863. [PubMed: 18825222]

8. Jurna M, Garbacik ET, Korterik JP, Herek JL, Otto C, Offerhaus HL. Anal. Chem. 2010; 82:7656. [PubMed: 20731373]

9. Rinia HA, Bonn M, Muller M, Vartiainen EM. Chem. Phys. Chem. 2007; 8:279. [PubMed: 17177224]

10. Chimento PF, Jurna M, Bouwmans HSP, Garbacik ET, Hartsuiker L, Otto C, Herek JL, Offerhaus HL. J. Raman Spectrosc. 2009; 40:1229.

11. Liu Y, Lee YJ, Cicerone MT. Opt. Lett. 2009; 34:1363. [PubMed: 19412273]

12. Oron D, Dudovich N, Yelin D, Silberberg Y. Phys. Rev. A. 2002; 65:043408.

13. Lim S-H, Caster AG, Leone SR. Phys. Rev. A. 2005; 72:041803.

14. Postma S, van Rhijn ACW, Korterik JP, Gross P, Herek JL, Offerhaus HL. Opt. Express. 2008; 16:7985. [PubMed: 18545508]

15. van Rhijn ACW, Offerhaus HL, van der Walle P, Herek JL, Jafarpour A. Opt. Express. 2010; 18:2695. [PubMed: 20174099]

16. Volkmer A, Book LD, Xie XS. Appl. Phys. Lett. 2002; 80:1505.

17. Selm R, Winterhalder M, Zumbusch A, Krauss G, Hanke T, Sell A, Leitenstorfer A. Opt. Lett. 2010; 35:3282. [PubMed: 20890360]

18. Rahav S, Mukamel S. Proc. Natl. Acad. Sci. U.S.A. 2010; 107:4825. [PubMed: 20185757]

19. Jurna M, Korterik JP, Otto C, Herek JL, Offerhaus HL. Phys. Rev. Lett. 2009; 103:043905. [PubMed: 19659356]

20. Du H, Fuh R-CA, Li J, Corkan LA, Lindsay JS. Photochem. Photobiol. 1998; 68:141.

21. Glauber RJ. Phys. Rev. 1963; 131:2766.

22. Brumer P, Shapiro M. Annu. Rev. Phys. Chem. 1992; 43:257. [PubMed: 18397166]

23. Frostig H, Katz O, Natan A, Silberberg Y. Opt. Lett. 2011; 36:1248. [PubMed: 21479047]

24. Ploetz E, Marx B, Gilch P. J Raman Spectrosc. 2011; 42:1875.

25. Zhang D, Slipchenko MN, Cheng J-X. J Phys Chem. Lett. 2011; 2:1248. [PubMed: 21731798]

Phys Rev Lett. Author manuscript; available in PMC 2013 July 28. 

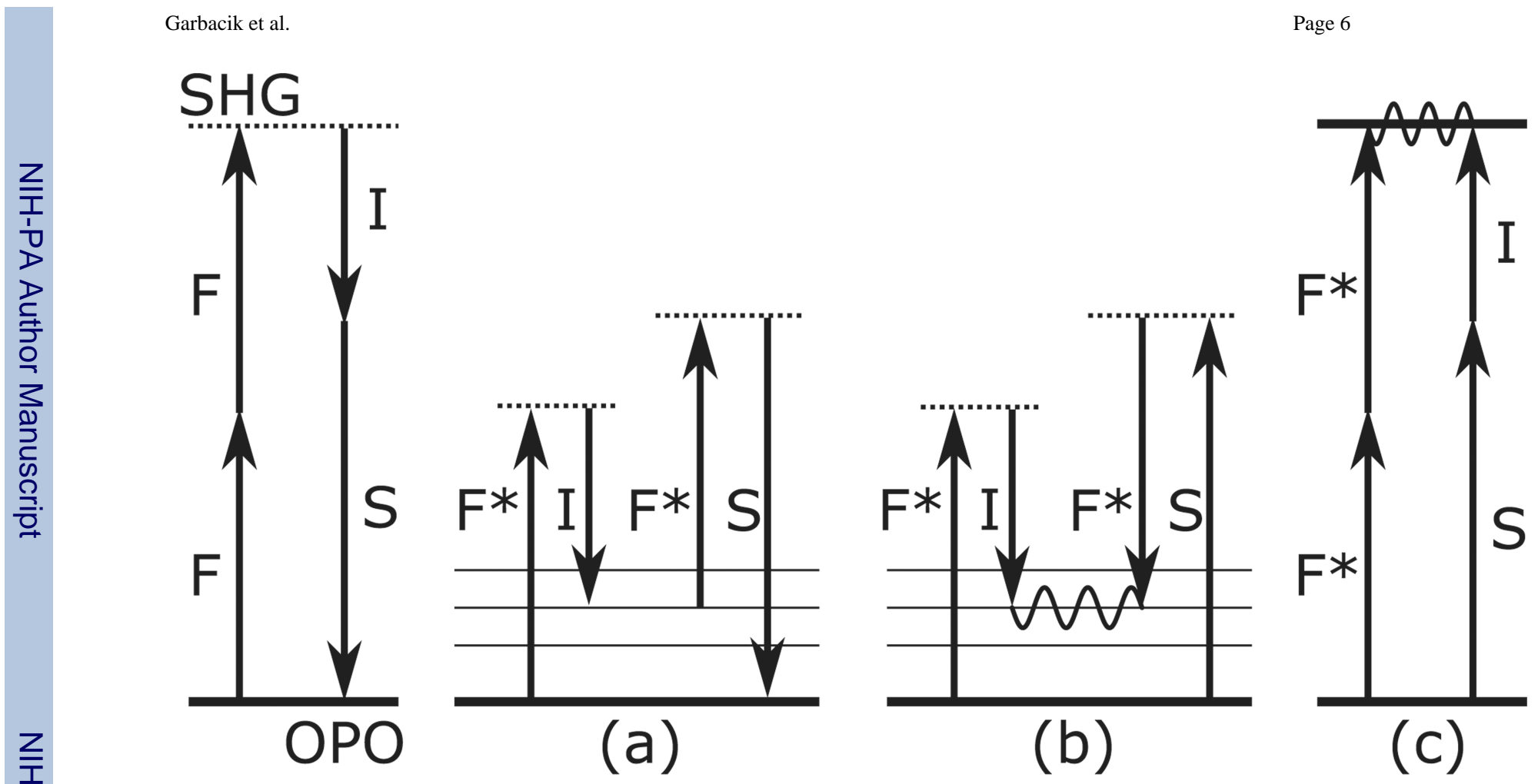

FIG. 1.

Energy level diagrams of the cascaded phase-preserving chain (left) and (a) parametric, (b) vibrational dissipative, and (c) electronic dissipative energy transfer processes in the molecule. $F=$ laser fundamental at $1064 \mathrm{~nm}, F^{*}=$ frequency modulated laser fundamental, $S=$ OPO signal, $I=$ OPO idler. Thick lines are electronic states, thin solid lines are vibrational levels, and dotted lines are virtual states. 
Forward CARS
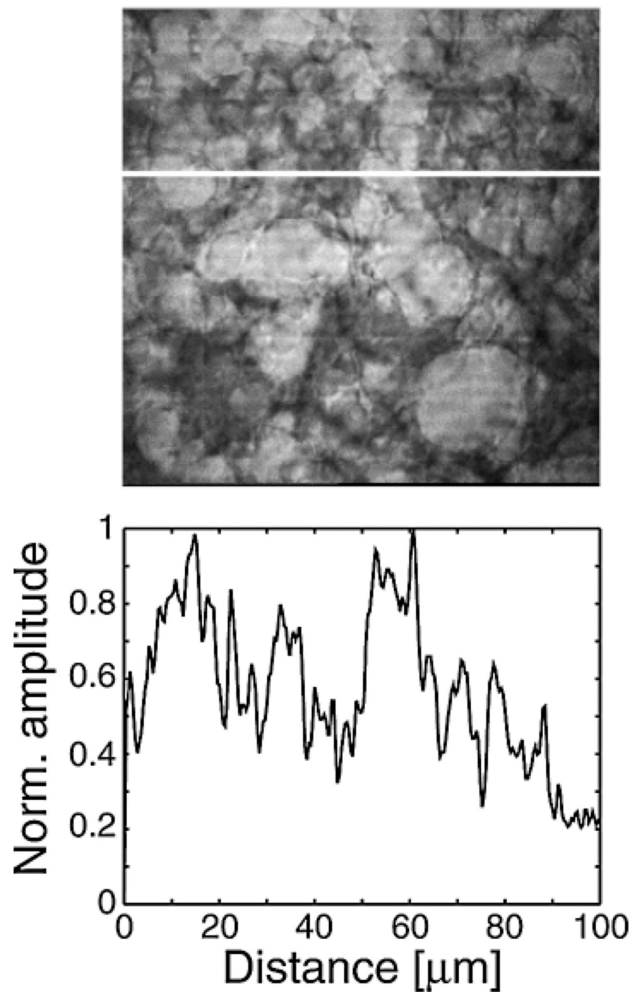

VPC-CARS
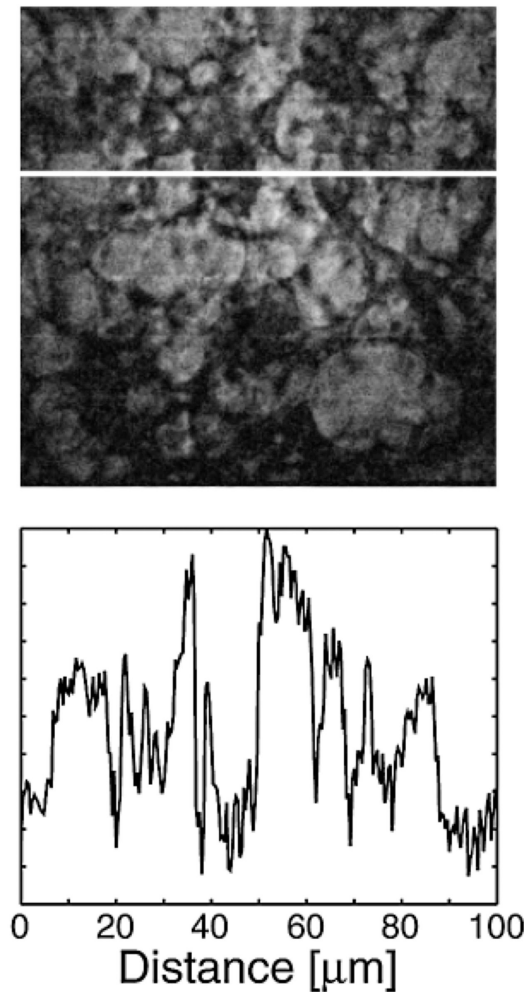

VMI
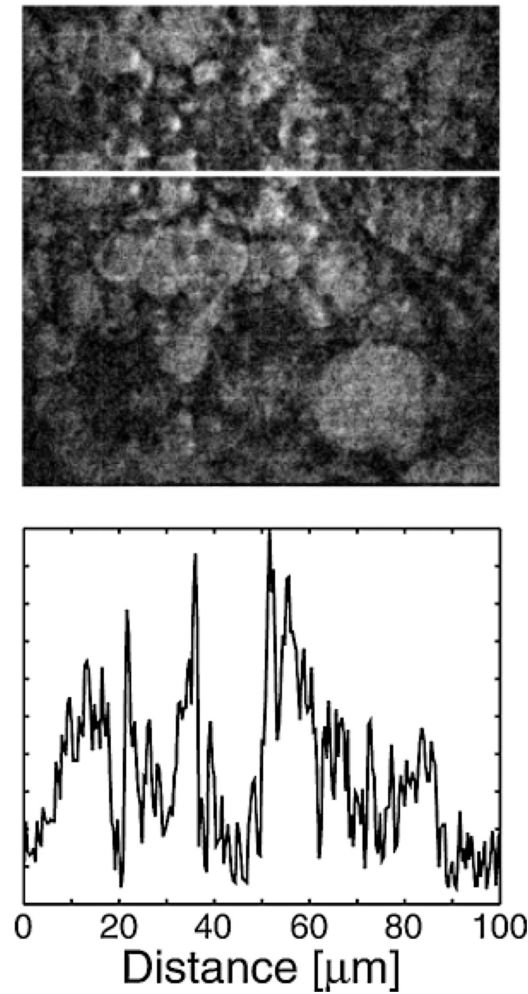

FIG. 2.

Mayonnaise images at $2845 \mathrm{~cm}^{-1}$ with CARS (left), VPC-CARS (center), and VMI (right), with intensity plots at the indicated line shown below. 


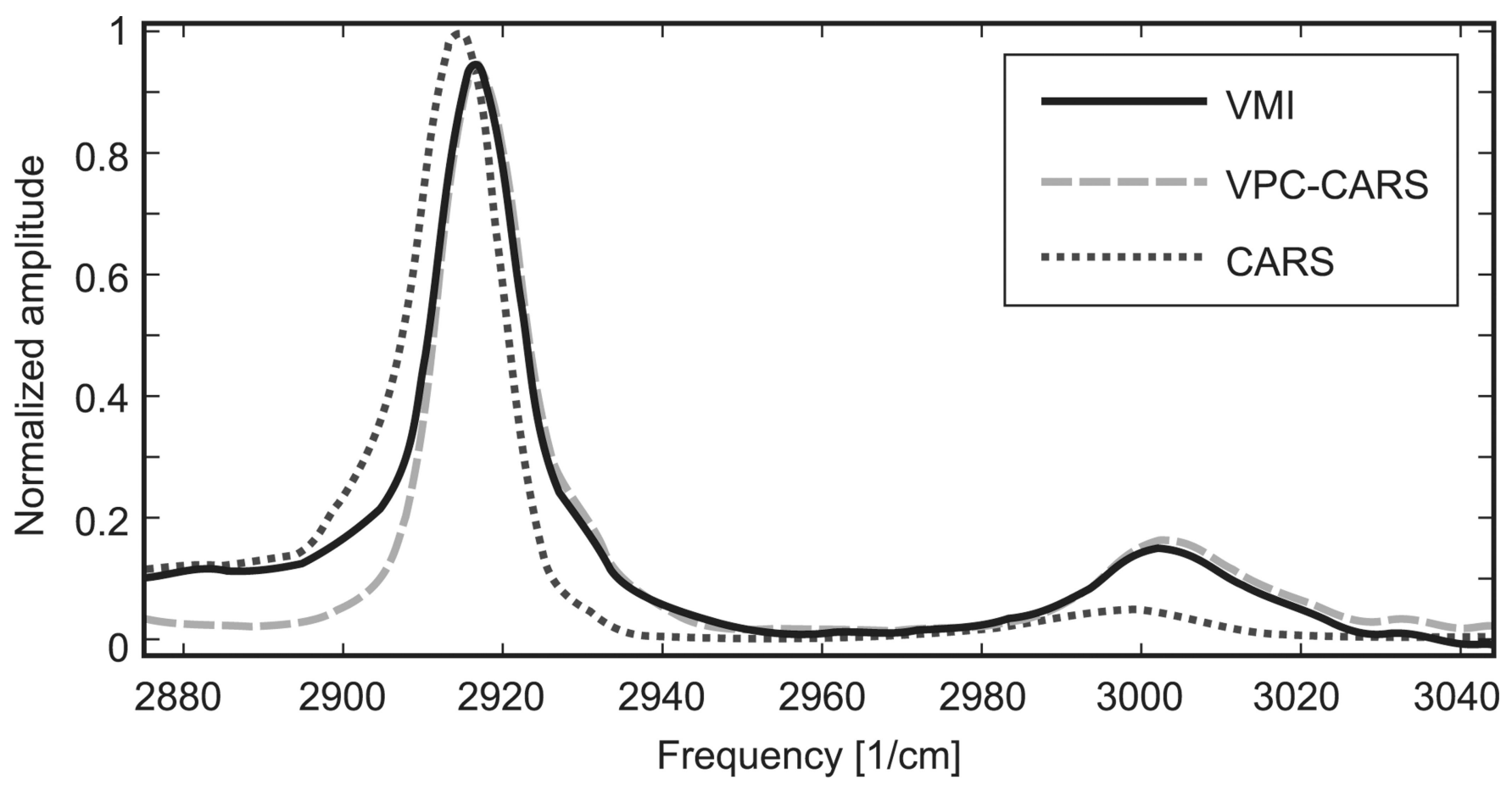

FIG. 3.

Vibrational spectrum of neat DMSO measured with CARS, VPC-CARS, and VMI. All spectra have been corrected for changes in optical power. Note that the CARS signal is actually an intensity rather than an amplitude. 

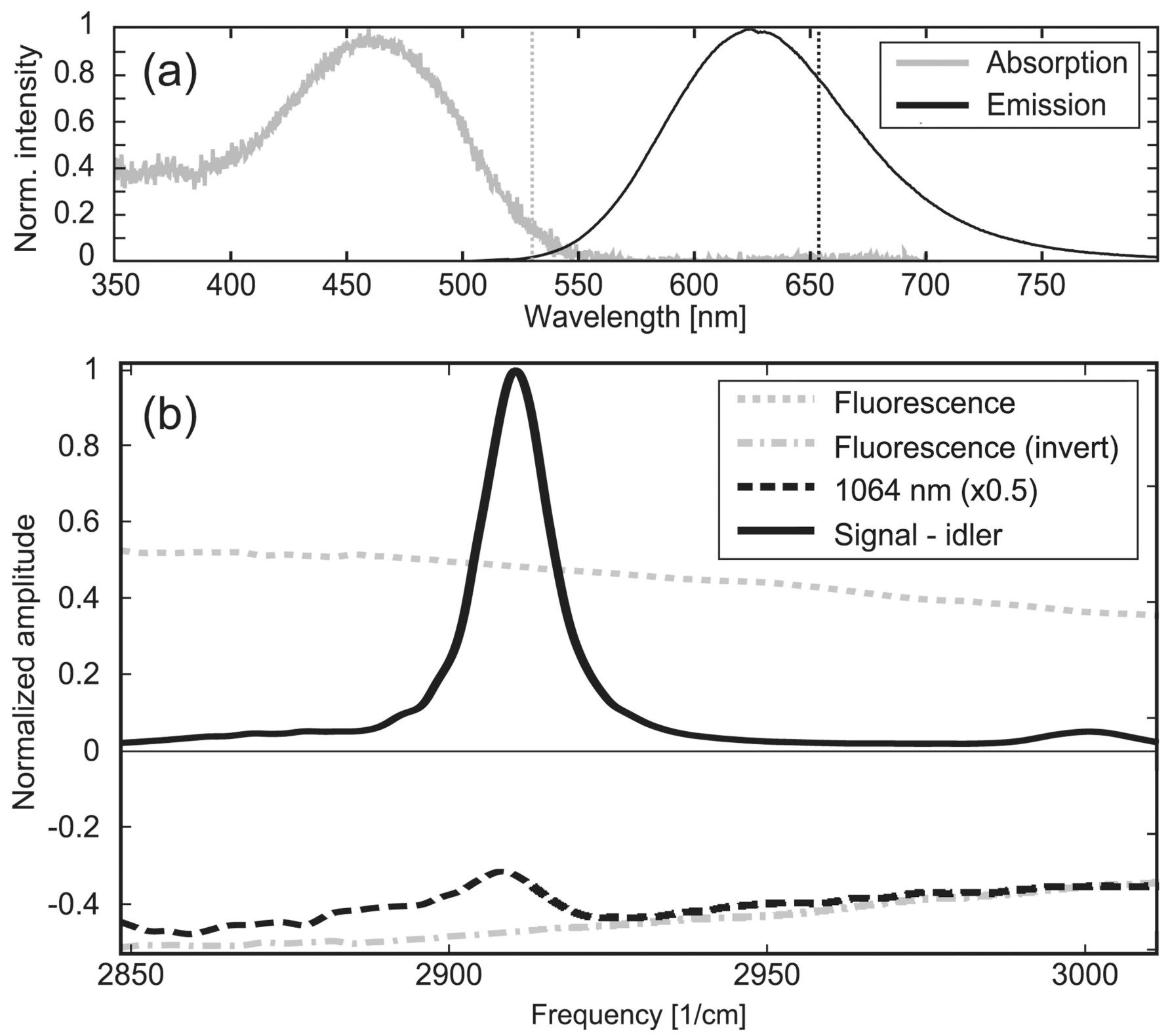

FIG. 4.

(a) Electronic spectrum of DCM-pyran. The vertical lines indicate (left) the SHG of the laser fundamental and SFG of the idler and signal and (right) the CARS wavelength. (b) Simultaneous background-free vibrational and electronic measurements of DMSO saturated with DCM-pyran. Inverting the fluorescence measurement associates it with an absorptive (loss) process, corresponding to the physical mechanism affecting the 1064-nm curve. None of the spectra have been corrected for optical power to illustrate the agreement between the fluorescence and 1064-nm profiles. 